\title{
GOBERNABILIDAD, DEMOCRACIA Y REFORMA DEL ESTADO: LOS DESAFÍOS DE LA CONSTRUCCIÓN DE UN NUEVO ORDEN EN BRASIL EN LOS AÑOS 90 *
}

\author{
Eli Diniz **
}

La escala e intensidad de los problemas que afectaron a la sociedad brasileña a partir de $\operatorname{los}$ años 80 configuran una crisis de múltiples dimensiones. La visibilidad de sus aspectos económicos, que se manifestaron en altos índices de inflación, fuerte endeudamiento externo, recesión y desempleo, oscureció la percepción de sus componentes políticos e institucionales, cuya principal expresión es la crisis del Estado. Una vez reconocida, esta última fue detectada fundamentalmente desde el ángulo del agotamiento de las condiciones de financiación del antiguo patrón de industrialización (Sola, 1993; 1994b). El desequilibrio fiscal, el desorden de las cuentas públicas y la inestabilidad monetaria fueron identificados como los factores propulsores del indispensable ajuste estructural introducido en la agenda pública a lo largo de los últimos diez años.

Aunque todo ello apunta hacia una cuestión importante, se trata de una visión parcial. La crisis fiscal tiene raíces profundas, razón por la cual no puede ser reducida a un fenómeno coyuntural, aunque su agravamiento esté relacionado con las dificultades específicas del actual contexto internacional. Entre sus fundamentos estructurales se sitúa el lento desgaste de la matriz político-institucional que moldeó el orden estatista bajo cuya égida evolucionó la industrialización sustitutiva de importaciones.

La crisis a la que se enfrenta el país no puede ser vista sólo como resultado de los impasses asociados a los sucesivos choques externos que marcaron la última década. El énfasis en los condicionamientos derivados de la dinámica del sistema internacional, tomados como variable determinante y exclusiva de explicación, conduce a análisis reduccionistas. De forma similar, las interpretaciones que privilegian las causas internas, sin tomar en consideración los requisitos de la inserción en un nuevo orden mundial, dan origen a visiones unilaterales, copiadas del supuesto de la autonomía de los Estados nacionales, tomados como entidades aisladas y, por lo tanto, inmunes a los efectos de la globalización. En contraposición a las aproximaciones dicotómicas, me parece más adecuada una perspectiva que integre los dos planos de análisis. Así, además de la dimensión externa, es preciso tener en cuenta los factores internos relacionados a la corrosión del orden precedente, dado el acrecentamiento de las tensiones entre sus elementos constitutivos.

Traducción del portugués: Ana Inés López-Accotto

(*) Publicado originalmente en la Revista Dados, V.38, nº 3, 1995

(**) Profesor de la Universidad Federal de Río de Janeiro.
Tal desgaste alcanzó a las formas prevalecientes de articulación Estado-sociedad, la dinámica de las relaciones capital-trabajo, el patrón de administración del conflicto distributivo y, sobre todo, la modalidad de relación entre los sectores público y privado. Bajo el impacto del desarrollismo de los años 70, el país experimentó cambios profundos, dando lugar a la estructuración de un sistema híbrido, caracterizado por la coexistencia de antiguas y nuevas configuraciones organizacionales e institucionales, un proceso que ya se encontraba en curso cuando hicieron eclosión las primeras señales de la reversión de tendencias en la esfera internacional ${ }^{1}$. En el contexto latinoamericano, el caso brasileño ilustra la extenuación del Estado como factor de contención de una sociedad civil en proceso de expansión y dotada de creciente densidad organizacional, razón por la cual el descompasamiento Estado-sociedad se sitúa en el meollo de los atolladeros actuales (Diniz y Boschi, 1994). La superposición de las crisis externa e interna puso en jaque la estrategia de la industrialización por sustitución de importaciones y el patrón de acción estatal que le sustentó (Diniz, 1991; 1993).

La complejidad de esa situación se torna aún mayor si consideramos los imperativos derivados del derrocamiento del régimen autoritario, junto a los requisitos de la consolidación de un orden democrático. Se observa, por lo tanto, el agotamiento simultáneo de un modelo dado de desarrollo económico, de sus parámetros ideológicos y del tipo de intervención estatal responsable de su implementación, dentro de un cuadro más general de reestructuración del orden político. De esta forma, la nueva agenda incluye, entre sus objetivos prioritarios, además de la estabilización económica y de la reinserción internacional, la institucionalización de la democracia.

La conciliación de esas metas no se reveló, por lo tanto, como una tarea simple ${ }^{2}$. A partir de la instauración de la Nueva República, en 1985, el intento de contener la inflación se hizo privilegiando estrategias coercitivas, con serias consecuencias para la priorización de las instituciones democráticas. De forma similar, las principales decisiones asociadas a la ejecución de las

1) En algunos de sus trabajos más recientes, Wanderley Guilherme dos Santos exploró el impacto de tales transformaciones en la dinámica social y política del Brasil contemporáneo, caracterizando lo que el autor denomina 'el hibrido institucional brasileño'. Ver, principalmente, Santos (1992; 1993).

2) En la vasta literatura sobre estabilización y reforma, algunos autores han enfatizado las tensiones entre los requisitos económicos de la estabilización y los requisitos políticos de la consolidación democrática. Ver, por ejemplo, Whitehead (1989). 
reformas liberalizadoras fueron confinadas a los círculos técnicos de la burocracia, reiterando la tendencia histórica de saltarse a la torera al poder legislativo. La búsqueda de una mayor eficacia y rapidez en la administración de la crisis fue reiteradamente interpretada mediante el recurso al estilo tecnocrático de gestión y de cierre burocrático de las decisiones, reforzando la centralización reguladora del Estado y acentuando el divorcio entre el Ejecutivo y el sistema de representación. De esta manera, la tensión entre las formas de alcanzar las metas refuerza la confrontación entre las prioridades de la agenda, convirtiéndose en parte constitutiva de la crisis del Estado, ya que compromete las bases de sustentación política del gobierno. Se reproduce un círculo vicioso de efectos perversos. Enfrentarse a la crisis requiere un Estado eficiente. Al mismo tiempo, la concepción dominante de la eficiencia estatal y la percepción de los medios para garantizar la anhelada eficacia reproducen los antiguos vicios de marginación de la política y de la primacía de los gobiernos tecnocráticos controlados por los círculos de especialistas. Tal modalidad de gestión pública, a su vez, tiende a producir el aislamiento del Ejecutivo, comprometiendo la racionalidad gubernativa.

Un factor adicional agrava este cuadro. La prioridad atribuida a los programas de estabilización económica y la crispación de los conflictos en torno a la distribución de recursos escasos terminan por expulsar ítems importantes de la agenda pública, tales como las reformas sociales. No sólo las posibilidades de crecimiento sino las perspectivas de atenuación de las desigualdades se vuelven metas cada vez más distantes. La urgencia del control de la inflación se acompaña del abandono de los proyectos igualitarios, que pasan a ser considerados bajo el ángulo de su extemporaneidad. Dentro de ese contexto, se planteó la exigencia de mayor inflexibilidad en la gestión de los recursos públicos, desaconsejándose, en nombre de un enfoque racional y no populista, cualquier postura favorable al aumento de los gastos sociales. El resultado fue el énfasis en una agenda minimalista, en franco descompasamiento con la ampliación de la participación y la diversificación de las demandas sociales.

El fracaso sucesivo de los ocho programas de estabilización, ejecutado por los dos primeros gobiernos de la Nueva República (19851992), amplió el abanico de problemas irresueltos, además de generar un desgaste grande de recursos y de la credibilidad de las instituciones. La percepción de la imposibilidad de abordar las cuestiones más apremiantes, como la inflación, la miseria y la violencia, se expresó por medio del desencanto y del sentimiento de impotencia política. Entre las consecuencias más notables, cabe mencionar la volatilidad del voto y la alta tasa de abstención electoral (Diniz, 1989b; Santos, 1992), en las elecciones nacionales y estatales, a lo largo de los diez últimos años, culminando en la elección de Fernando Collor, en 1989. Ganó peso la idea de que los problemas eran insolubles, sin salida palpable, dadas las condiciones reales del país. Los escándalos que rodearon el funcionamiento del Congreso acentuaron aún más ese descreimiento generalizado en la eficacia de las instituciones.

\section{GOBERNABILIDAD/INGOBERNABILIDAD: EL RESULTADO DE UNA TRANSICIÓN INCONCLUSA}

Bajo el efecto de las condiciones arriba enfatizadas, la recién instaurada democracia brasileña sería percibida en forma creciente como ingobernable. De este modo, el tema de la gober- nabilidad asciende al primer plano del debate nacional, situándose como central en el diagnóstico de la crisis y en la formulación de las estrategias para hacerle frente. La capacidad de gobierno y la eficacia en la conducción de los negocios públicos emergen como aspectos estrechamente interrelacionados. Reintroducido en el vocabulario político brasileño, el término ingobernabilidad se convierte en un símbolo de la falta de competencia del país para lidiar con sus problemas, emitiendo señales de que nuestros líderes y nuestras instituciones no estarían a la altura de nuestras necesidades actuales.

Sin embargo, al volverse un item prioritario de la agenda pública, la cuestión de la ingobernabilidad adquiere diferentes connotaciones, significando tanto la presión exagerada de participación y de demandas, como el poder excesivo del Congreso e incluso el exceso de prerrogativas de la autoridad pública asociado a la escasez de mecanismos de control, traduciéndose en el síntoma de clientelismo, corrupción y despilfarro ${ }^{3}$. La multiplicidad de interpretaciones fue incentivada por la politización de las discusiones, enfatizándose uno u otro de los significados en función de los intereses implicados en la disputa política. La diversidad de sentidos terminó por vaciar el término, desdibujando su valor explicativo. Impregnado aun de connotaciones ideológicas de tono conservador por su utilización en el debate internacional, pasó a ser visto como instrumento del juego de poder que se desplegaba en la esfera mundial.

La constatación de la interferencia de factores ideológicos en la formulación de diagnósticos y terapias para enfrentar la llamada crisis de gobernabilidad debe, sin embargo, ser relativizada, ya que se trata de un dato inherente al proceso político. No se puede descartar la pertinencia del problema como consecuencia del exceso de acepciones conferidas por las distintas posiciones en conflicto. He aquí un punto que merece ser resaltado.

A despecho de la politización del debate, la importancia de la discusión teórica en torno a la cuestión de la gobernabilidad se clarifica si consideramos las paradojas existentes en la transición brasileña. Si, por un lado, ese proceso puede ser considerado exitoso en la medida en que condujo a la implantación de un régimen democrático pleno consagrado por la Constitución de 1988, por otro, la construcción de un nuevo orden no se basó en una amplia y profunda renovación político-institucional (Diniz, 1985). Como es sabido, esa transición se caracterizó por un alto grado de continuidad en el plano de las élites y de las instituciones, persistiendo rasgos del modelo estatista, entre ellos la hipertrofia del Ejecutivo y la fragilidad del sistema de partidos. Aspectos coyunturales ligados a la modalidad de intervención estatal para afrontar la crisis, exacerbaron la asimetría de los Poderes, estrechando los márgenes para el ejercicio de una acción autónoma por parte del Legislativo. A través de una dinámica de realimentación recíproca, tal desequilibrio contribuyó a la pérdida de credibilidad de las instituciones, reduciendo la legitimidad y la autoridad del gobierno en su conjunto (Diniz, 1989a).

Finalmente, si se tiene en cuenta la centralidad de la solidez institucional para la creación de las condiciones favorables para una mayor eficacia de la acción gubernamental en lo que respecta a la búsqueda de salidas para las actuales dificultades y

(3) En el texto, Ingobernabilidad: desagregando el Argumento, Marcus Andre B.C. de Melo (1994) discute de forma bastante exhaustiva las variadas significaciones atribuidas al concepto de gobernabilidad en Brasil a lo largo de la última década. 
para la ejecución de una nueva estrategia de desarrollo, no se puede descartar la relevancia de ciertas indagaciones sugeridas por el debate anterior. Cabe, por lo tanto, retomar la discusión. El presente texto tiene por objetivo profundizar en la reflexión sobre el concepto de gobernabilidad, su sentido analítico, sus implicaciones teóricas y la evaluación de su utilidad en el estudio de la producción de políticas públicas en las esferas federal y local. Además de ese esfuerzo, se procura establecer las distinciones entre gobernabilidad y governance, como aspectos complementarios de una cuestión crucial, como es la eficacia de la acción estatal, a la luz de los desafíos generados por los cambios que afectaron a la sociedad brasileña en las dos últimas décadas.

El argumento central se propone examinar la reforma del Estado en conexión con la problemática de la consolidación democrática, procurando evitar las trampas derivadas de las dos principales dicotomías que enturbian los análisis más generalizados. Por un lado, la polaridad Estado-mercado, que contrapone una visión maximalista (presa del paradigma estatista) a una concepción minimalista, responsable de la idealización del mercado. Por otro, la dicotomía entre racionalidad gubernamental e imperativos democráticos, aquella constituida por una lógica concentracionista y discrecional, en contraste con una dinámica descentralizadora y competitiva del juego democrático.

\section{GOVERNANCE: CAPACIDAD DE GOBIERNO Y EFICACIA ESTATAL}

Las sucesivas reformulaciones del concepto de gobernabilidad, en los términos indicados en la sección anterior, al eliminar los aspectos reduccionistas y el sesgo elitista que reducían su alcance, lo hicieron incompatible con los imperativos del fortalecimiento de las instituciones democráticas. Al mismo tiempo, las múltiples significaciones que se le atribuyeron terminaron por afectar su eficacia analítica. Además, la nueva acepción se confundía con el concepto de governance. Tal superposición es fruto de la imprecisión conceptual que ha caracterizado a la producción en ese área.

El término governance fue introducido en el debate público internacional como resultado de una reflexión dirigida principalmente por el Banco Mundial, buscando profundizar el conocimiento de las condiciones que garantizan un Estado eficiente. Dicha preocupación desplazó el foco de atención de las implicaciones estrictamente económicas de acción estatal, hacia una visión más abarcadora, que incluyera las dimensiones sociales y políticas de la gestión pública. En sentido amplio, la capacidad gubernativa no sería más evaluada en función sólo de los resultados de las políticas gubernamentales, pasando a significar la forma por la cual el gobierno ejerce su poder. Según la formulación del Banco Mundial, governance "is the process by which authority is exercised in the management of a country's economic and social resources" implicando también "the capacity of governments to design, formulate and implement policies and discharge functions" ${ }^{*}$. De esta manera, la cuestión de los procedimientos y prácticas gubernamentales en la consecución de

* En inglés en el original (N. de T.).

(4) Tal formulación aparece en el documento Governance and Development (Banco Mundial, 1992a, sus metas adquiere relevancia, incluyendo como objeto de análisis cuestiones como la forma institucional del proceso de toma de decisiones, la articulación público-privado en la formulación de las políticas públicas o incluso la apertura mayor o menor hacia la participación de los sectores interesados o de distintas esferas de poder (World Bank, 1992b).

Otra tendencia de las agencias multilaterales fue el empleo del término en un sentido más normativo, refiriéndose a las expresiones good governance o good government, para enfatizar el buen desempeño del gobierno en base a criterios como justicia, igualdad y derechos humanos. En realidad, lo que está implícito en esas formulaciones es la asociación entre alta capacidad gubernamental y democracia política. El supuesto es la existencia de una estrecha relación entre el régimen democrático y las condiciones que garantizan good governance. Cabe resaltar que, desde esta perspectiva, el concepto ve reducirse su capacidad de abstracción. Varios estudios han demostrado la ausencia de fundamento empírico de la pretendida relación entre régimen político y éxito en la implementación de políticas de ajuste o de desarrollo (Remmer, 1986 y 1990; Lefwich, 1993; Geddes, 1994; Maravall, 1994). En otros términos, los análisis más recientes muestran que no hay evidencias de que los casos de éxito o de fracaso estén asociados al régimen político en vigor.

Finalmente, la discusión actual en torno al concepto de governance traspasa los aspectos estrictamente ligados al modus operandi de las políticas, englobando cuestiones más amplias relativas a patrones de coordinación y de cooperación entre actores sociales y políticos (Melo, 1994). Según esa línea de análisis, las condiciones de governance configuran un sistema que comprende la totalidad de los acuerdos institucionales que coordinan y regulan las transacciones en el interior y más allá de las fronteras de un sistema económico (Hollingsworth, Schmitter y Streeck, 1993 apud Melo, 1994).

Teniendo en cuenta las dificultades señaladas, me parece importante recuperar algún grado de especificidad de los conceptos, marcando simultáneamente sus diferencias. De esta manera, cabe mantener la referencia a la gobernabilidad para caracterizar las condiciones institucionales y sistémicas más generales bajo las cuales tiene lugar el ejercicio del poder en una sociedad dada, tales como la forma de gobierno, las características de los sistemas de partidos y electoral, entre otras. No hay fórmulas mágicas capaces de garantizar la gobernabilidad, que puede ser producida a partir de diferentes combinaciones institucionales. Por otro lado, para analizar cuestiones ligadas a la capacidad de acción del Estado, en la implementación de las políticas públicas y en la consecución de las metas colectivas, me parece pertinente utilizar el término governance, más apropiado para abordar la dimensión participativa y plural de la sociedad brasileña, que se expandió de forma considerable a partir de los años 70.

Como se ha resaltado reiteradamente, el diagnóstico acerca de las dificultades presentes en el enfrentamiento de los problemas críticos del país no puede dejar de lado la cuestión de la crisis del Estado. Uno de los componentes básicos de su vulnerabilidad es una eficacia operacional decreciente y una total incapacidad de hacer valer sus decisiones. La hiperactividad decisoria asociada a la impotencia ejecutiva y a la inoperancia estatal caracterizan la debilidad del poder público en Brasil. Se trata de un problema crucial que no puede quedar fuera de los 
análisis, so pena de no avanzar en el diagnóstico y en la formulación de las salidas a las dificultades actuales. En este sentido, el meollo de la reforma del Estado reside en la recuperación de la capacidad de gobierno, profundamente comprometida como reflejo de factores de orden histórico-estructural y coyuntural.

En esa línea de reflexión, governance se refiere a tres dimensiones esenciales. La primera es la capacidad de mando y de dirección del Estado, tanto internamente, de cara al conjunto de la maquinaria estatal y de la sociedad, como externamente, vis$a$-vis la inserción del país en el sistema internacional. La segunda dimensión relevante es la capacidad de coordinación del Estado entre las distintas políticas y los diferentes intereses en juego. Finalmente, la capacidad de implementación es otro aspecto crucial, resaltado, además, por varios análisis como elemento esencial de la crisis del Estado en su manifestación actual $^{5}$.

La cuestión de la capacidad de mando del gobierno no ha sido suficientemente analizada. Significa no sólo asumir la dirección efectiva del proceso de producción de políticas públicas realizado por el conjunto del aparato estatal, sino definir y ordenar prioridades, garantizando su continuidad a lo largo del tiempo. Se trata de la capacidad de definir las estrategias generales de acción, las grandes directrices que guían a las decisiones gubernamentales. La inexistencia de una dirección general que integre las diversas fases del proceso decisorio a determinadas metas globales abre el camino para una actuación desarticulada y fragmentada, sometida a la lógica de metas e intereses contradictorios.

De manera complementaria, la capacidad de coordinación del Estado se refiere a la integración entre las diferentes áreas de gobierno, dirigida a garantizar la coherencia y consistencia de las políticas gubernamentales. Implica también la capacidad de ajuste entre programas focalizados y programas sectoriales, por un lado, y políticas de mayor alcance y abarcabilidad, por otro. Finalmente, coordinación significa someter la lógica de los intereses en juego a un sistema integrado capaz de compatibilizar las diferentes visiones. No se trata, pues, de eliminar el conflicto, sino de administrarlo de forma eficiente, es decir, de forma compatible con la racionalidad gubernativa, que es definida en función de un proyecto colectivo.

En ese aspecto, nunca está demás enfatizar la importancia de concebir la acción del Estado como un proyecto global. En el debate actual, existe una tendencia a entender la eficacia estatal como derivada del grado de claridad y precisión por medio del cual se definen las metas a alcanzar. Se trata, en realidad, de una concepción estrecha y difícilmente compatible con el funcionamiento de un orden democrático. Es preciso no olvidar que la democracia implica justamente la problematización del proceso de constitución de los fines, necesariamente múltiples e incluso antagónicos, sobre todo en una sociedad compleja y diferenciada como la brasileña ${ }^{6}$. Agregar y hacer compatibles tales fines requiere procedimientos continuos de negociación. La visión propuesta aquí se contrapone a la perspectiva estatista y a la noción de autonomía del Estado en cuanto capacidad de situar-

(5) Ese uso de la noción de governance tiene puntos de contacto con el análisis de Leila L. Frischtak en dos textos recientes (1994a; 1994b). Agradezco a la autora el acceso a esos textos, que presentan una discusión interesante de los conceptos de gobernabilidad y governance tal como son tratados en la literatura más reciente sobre ajuste y reforma.

(6) Ese aspecto es enfatizado por Reis en varios de sus análisis sobre la cuestión de la gobernabilidad en contextos democráticos (Reis, 1993; 1994a; 1994b; 1995). se por encima de las presiones, que implicaría, en última instancia, la definición de las metas por las propias élites estatales, disociadas de la esfera en que se manifiestan sobre todo los intereses más dispersos o menos organizados de la sociedad'. De forma similar, es preciso recuperar la ética del interés público, una cuestión crecientemente descartada como irrelevante por su posible proximidad a la idea de interés nacional, a su vez concebida como incompatible con la agenda de la modernidad dominada por la noción de globalización. En contraste, para no ser una expresión vacía o puramente retórica, el interés público debe reflejar la acción eficiente de las instancias de agregación en las que se produce la compatibilización de aquello diversificado y contradictorio de las demandas sociales. En otros términos, se traduce en un proyecto colectivo, que representa la capacidad del sistema político en general y de las élites gobernantes en particular de convivir con el disenso y el conflicto, no mediante el esfuerzo de anularlos, sino a través de la disposición para la negociación y el compromiso.

Cabe resaltar que la garantía de la autonomía estatal respecto a las presiones clientelares o particularistas depende, en gran parte, del ejercicio de la capacidad de coordinación y liderazgo del Estado. Sin esta supervisión, lo que tiende a predominar es la dinámica de soluciones inspiradas en la racionalidad de corto plazo o en la maximización de los beneficios individuales. La desprivatización del sector público, evitando su captura por intereses privados, requiere el refuerzo de la autonomía del Estado sin, por ello, eliminar el juego democrático. Al contrario, es bajo condiciones de ausencia de coordinación como la extensión de la privatización puede amenazar la eficiencia de las políticas estatales.

Por último, la capacidad de implementación es otro aspecto crucial para la recuperación de la eficacia de la acción estatal. Para ello, el Estado debe ser capaz de movilizar los recursos técnicos, institucionales, financieros y políticos necesarios para la ejecución de sus decisiones. En primer lugar, es preciso que tales recursos estén disponibles; en el caso de que no existan o no sean consistentes con las decisiones, se vuelve imprescindible crearlos. En segundo lugar, se debe considerar los recursos junto a la competencia técnica y la excelencia del equipo administrativo. Tales aspectos son fundamentales, pues la calidad del personal administrativo, la existencia de un cuerpo técnico de alto nivel, el refuerzo del sistema meritocrático y de las normas universalistas son condiciones para garantizar el carácter público del Estado. El clientelismo y el particularismo son contradictorios con la noción de dominio público, ya que ésta presupone un tratamiento igualitario, sin distinciones, privilegios o regalías basados en redes de relaciones y lealtades personales.

Además de eso, la existencia de un acervo de informaciones, de una base de datos almacenados de forma eficiente constituye también un componente necesario de la eficiencia operativa del Estado.

Aunque importantes, los aspectos arriba señalados no son, sin embargo, suficientes para el éxito de la ejecución. Es preciso también tomar en consideración las condiciones de sustentación política de las decisiones. La naturaleza eminentemente política de la ejecución requiere, por lo tanto, una estrategia que

(7) Ese argumento encuentra respaldo en una línea teórica que resalta los aspectos igualitarios de la noción de democracia asociativa, la cual, a su vez sitúa las diferentes concepciones de bien común como un importante referente del proceso decisorio en un orden democrático (Cohen y Rogers, 1992). 
mantenga abiertos los canales de comunicación con la sociedad y el sistema representativo, incluyendo los partidos políticos, cuya relevancia no puede ser ignorada.

Finalmente, el enfoque exclusivamente técnico acerca de las condiciones de éxito de las políticas estatales no es adecuado respecto al ejercicio de la capacidad de gobierno en un contexto democrático. Esa visión siempre predominó en Brasil, llevando a confundir la eficacia operativa con concentración del poder de decisión en agencias clave. Tal distorsión, lejos de garantizar la eficiencia, llevó, frecuentemente, a la pérdida de accountability. Por el contrario, la obligación de la presentación de cuentas al público y a las autoridades competentes, la existencia de mecanismos de recaudación y de controles mutuos constituyen un factor favorable al éxito de la ejecución, en la medida en que permiten evitar la apropiación del aparato por parte de intereses privados, el despilfarro, así como la desviación de recursos para fines no previstos. En fin, la transparencia de los procesos de gestión y de ejecución es un requisito de la acción del gobierno en un régimen democrático. En sí mismo ese factor no representa una amenaza a la consecución de las políticas, sino que contribuye a aumentar la eficacia gubernamental.

Concluyendo, los puntos discutidos aquí apuntan a la necesidad de la reforma del Estado como condición para abordar la presente crisis a la que se enfrenta el país y para la consecución de una nueva estrategia de desarrollo. En este sentido, ciertos aspectos de la reforma institucional se vuelven relevantes al tener como objetivo el alcanzar simultáneamente las metas de refuerzo de la eficiencia del Estado y de perfeccionamiento de las instituciones representativas. Para garantizar el control público sobre las decisiones no basta el abandono de estrategias de acción coercitivas por parte de la burocracia gubernamental y sus sustitución por estrategias cooperativas. Es preciso que se fortalezcan los partidos y el poder legislativo. La persistencia del divorcio entre Ejecutivo y Legislativo en la conducción de las políticas nacionales y en la gestión administrativa puede conducir a la reproducción de negociaciones encerradas en la burocracia, sin un sistema eficaz de controles mutuos.

Superar el actual impasse representado por la ausencia de tratamiento de problemas cruciales como la inflación, la desigualdad y la exclusión social, requiere la ruptura con el enfoque tecnocrático y con una postura neoliberal. El primero, al privilegiar el aislamiento de las élites estatales y la primacía del saber técnico, conduce a una despolitización artificial de los procesos de formulación y ejecución de políticas. La segunda, al enfatizar unilateralmente la reducción del Estado, ignora la estrecha relación entre la revitalización del aparato estatal y el éxito de sus políticas. Contraponiendo las virtudes del mercado a los vicios del Estado, la posición minimalista se ve enriquecida por una visión de juego de suma cero, incapaz de percibir la relevancia del perfeccionamiento de la intervención gubernamental para que el mercado funcione de forma eficiente y en beneficio de un proyecto colectivo. Superar los obstáculos ligados a la dicotomía Estado-mercado implica, por lo tanto, un nuevo patrón de gestión pública, entendida como la capacidad de ejecutar una estrategia de reformas en conexión con la dinámica de los intereses sociales ${ }^{8}$.

(8) Para un análisis de la relación entre el éxito de las políticas de ajuste y distintos patrones de interación Estado-sociedad, ver Locke (1995).

\section{CONSIDERACIONES FINALES}

A lo largo de este artículo, he procurado revisar los conceptos de gobernabilidad y governance, explorando sus conexiones con la reforma del Estado, bajo condiciones de funcionamiento del régimen democrático. El núcleo de la argumentación privilegió la complejidad de la agenda pública después del derrocamiento del autoritarismo, en la que se destacan dos prioridades básicas: la administración de la crisis económica y la consolidación de un orden democrático.

La dificultad de conciliación de esas metas se reveló superior a los recursos y estrategias de acción de los principales actores en juego, constituyéndose en una de las razones del impasse que el país enfrenta en la actualidad. La eficacia en la administración de la crisis y la revigorización de las instituciones democráticas fueron planteados como objetivos contradictorios, perdiéndose de vista la perspectiva de largo plazo, por medio de la búsqueda de beneficios inmediatos en la conducción de la cotidianeidad de la vida política. La interpretación de la eficiencia estatal en términos de aislamiento burocrático acentuó la tendencia histórica a la constitución de un Ejecutivo fuerte, que no sólo concentra el poder de decisión, sino que relega al Legislativo a una posición periférica desde el punto de vista de la formulación de las políticas públicas relevantes. Predominó la visión tecnocrática de la gestión económica, confiriéndose todo el poder al llamado núcleo duro del Estado, constituido por una élite de especialistas, informados por una subcultura marcada por la primacía de la racionalidad técnica, en la que la descalificación de la política y de la actividad partidaria es la tónica. Percibida sistemáticamente como fuente de irracionalidad y de presiones espúreas, la arena política es reducida, en la práctica, a una fuerza que debe ser neutralizada, como tiende a ocurrir cuando una determinada cuestión es vista desde la óptica del juego de suma cero. De esta forma, la tensión entre las formas de realizar las prioridades de la agenda es parte constitutiva de la crisis del Estado ya que, según la óptica dominante, atender al requisito de la eficiencia implica aislar al Ejecutivo y comprometer las bases de sustentación política del Gobierno.

En estrecha relación con esa problemática se sitúa el segundo aspecto central en el diagnóstico que aquí se propone sobre la crisis del Estado. La debilidad del poder estatal no reside en una supuesta parálisis decisoria, sino en la paradoja de una hiperactividad decisoria en contraste con la debilidad ejecutiva y la incapacidad de hacer valer las decisiones tomadas. Lejos de retraerse, el Ejecutivo ejerce al máximo su poder regulatorio e interventor, definiendo y supervisando la pauta del Legislativo y también controlando el ritmo de tramitación de los proyectos y propuestas, imponiéndose de hecho como el principal centro de producción legal del país. El monopolio del poder de iniciativa y la supremacía del Ejecutivo, mediante procedimientos como el uso indiscriminado de medidas provisorias, a su vez, acentúan la debilidad histórica de los partidos y del Congreso, induciéndolos a formas de comportamiento irresponsables.

En contraposición, la capacidad de ejecución de las decisiones aprobadas por los altos escalones burocráticos se encuentra seriamente comprometida. Los obstáculos a la ejecución, imponiendo fracasos parciales o totales sucesivos a los programas gubernamentales, tienden a ser sistemáticamente percibidos como el resultado de la acción de fuerzas externas desestabilizadoras. Tal interpretación inspira formas de reacción que 
refuerzan el poder discrecional del Ejecutivo. Las tentativas de eludir este tipo de dificultades y escapar del inmovilismo asociado al déficit de ejecución recrean las condiciones de aislamiento burocrático, reforzando las barreras contra presiones no deseadas, en un proceso de realimentación recíproca. El confinamiento de la tecnocracia en agencias protegidas de los embates de la política competitiva han sido la terapia tradicionalmente concebida para afrontar ese orden de problemas (Haggard e Kaufman, 1993; Sola, 1994a, pp.157-9).

La incapacidad demostrada por los tres primeros gobiernos de la Nueva República en la administración de la crisis económica y social del país llevó a que la cuestión de la crisis de gobernabilidad asumiese el primer plano de la agenda pública. La percepción de la incapacidad de enfrentarse a problemas como la inflación, el déficit público, la desigualdad y la exclusión social, entre otros, generó una creciente pérdida de credibilidad del gobierno y de las instituciones políticas en general, corroyendo los factores capaces de garantizar la legitimidad del sistema político.

Los innumerables análisis y los sucesivos diagnósticos acerca del estado de ingobernabilidad detectado como el núcleo de nuestras dificultades presentes incurrieron, por tanto, en algunos equívocos básicos, responsables de su insuficiente alcance explicativo. Tres tipos de deficiencias estrechamente interrelacionadas se hicieron relativamente recurrentes. En primer lugar, se observa de forma bastante difundida una visión marcada por un sesgo reduccionista, que concentra la interpretación en un aspecto unidimensional, remitiendo la explicación a una causa determinada, por ejemplo la sobrecarga de demandas. Un segundo tipo de enfoque está influido por un sesgo tecnocrático, que define ingobernabilidad en términos de inercia decisoria, avalada como consecuencia del exceso de presiones exógenas. Finalmente, se puede notar la presencia de una perspectiva elitista, que centra su diagnóstico en los efectos perversos del activismo social y en el desbordamiento de la participación política más allá de los límites definidos por la funcionalidad del sistema.

En tales aproximaciones subyace la concepción tecnocrática de eficiencia estatal, que sitúa la fuerza y la autonomía del Estado como antídotos contra los desequilibrios apuntados, entendiendo esos requisitos en términos de aislamiento de los núcleos tecnoburocráticos, cerrando el círculo decisorio y la primacía del conocimiento técnico. La perspectiva centralizadora, a su vez, refuerza la falacia tecnocrática, basada en la suposición de que los conflictos en torno a las políticas de carácter más polémico pueden ser resueltos hurtándolos a las instancias políticas, confinándolas al interior de la burocracia (Haggard, 1995; Ducatenzeiler e Oxhom, 1994). Además de eso, los análisis mencionados coinciden en privilegiar soluciones que implican reducir la competitividad del sistema político, preconizando artificios de ingeniería institucional, sobre todo cambios en las legislaciones electoral y partidaria, para corregir las alegadas distorsiones en el ámbito de la participación y de la representación. La expansión de conflictos, la sobrecarga de demandas, la fragmentación partidaria, la exacerbación de las expectativas y la diversificación de las presiones serían los principales aspectos característicos de un orden social ingobernable.

Esa línea de argumentación no toma en consideración que en Brasil, a pesar de tener un sistema poliárquico, no se verifican las condiciones de una ciudadanía plena, observándose la existencia de una amplia parcela de la población que vive bajo condiciones de desinstitucionalización social y política (O’Donnell,
1993; Santos, 1992), o sea, la esfera poliárquica se superpone a grandes espacios institucionalmente vacíos, en los cuales lo que prevalece es la ausencia de derechos y la falta de acceso a un orden legal, lo que reduce o incluso inviabiliza el ejercicio de la ciudadanía. Además, el razonamiento conduce a un impasse analítico, pues la explosión de demandas y la saturación de la agenda serían la resultante de un aspecto paradójico de la dinámica democrática. Por un lado, como sabemos, la democracia implica extensión de derechos y ampliación de la participación. Por otro, el ejercicio pleno de tales prerrogativas acarrearía una creciente erosión de la capacidad del gobierno, produciendo efectos perversos de debilitamiento de las instituciones democráticas. Poner en marcha mecanismos restrictivos orientados a la contención de demandas y reducción de la competencia política sería la salida.

Según la perspectiva desarrollada aquí, las crisis de gobernabilidad y del Estado son indisociables y deben ser estudiadas en sus múltiples dimensiones. Aspectos conyunturales ligados a los efectos económicos y políticos de la crisis internacional se asocian a la dinámica interna de corrosión del orden estatista bajo el cual se dio la construcción del capitalismo industrial en el país, generando un cuadro complejo y multifacético y haciendo inocua la búsqueda de explicaciones unicausales.

En segundo lugar, las estrategias de enfrentamiento a crisis de esta naturaleza no pueden perder de vista la meta de la consolidación democrática. En ese aspecto, la pregunta central que se intentó responder se refiere a cómo compatibilizar la eficiencia del Estado y el perfeccionamiento de la democracia. Paralelamente, la necesidad de refinamiento del análisis se tradujo en un énfasis en la dimensión política, junto a la dimensión técnica. La noción de eficacia del Estado se trabajó englobando no sólo cuestiones ligadas a la competencia y la eficiencia de la maquinaria estatal, sino también aspectos asociados a la sustentabilidad política de las decisiones y a la legitimidad de los fines que se pretende alcanzar por medio de la acción gubernamental. Aumentar los niveles de gobernabilidad de un orden democrático implica no sólo mejorar el desempeño del aparato burocrático, sino ampliar la responsabilidad del Estado en cuanto a las metas colectivas y a las demandas sociales existentes. De modo similar, la noción de gobernabilidad fue examinada en su aspecto pluridimensional, abarcando los instrumentos institucionales, los recursos financieros y los medios políticos de ejecución de las políticas formuladas. Finalmente, se trató la reforma del Estado teniendo en cuenta la meta de maximizar las condiciones de governance del sistema estatal, que implica la expansión de la capacidad de dirección, de coordinación y de implementación del Estado, cuestión que, en última instancia, requiere una estrategia política para conducir acuerdos y alianzas, articulando arenas de negociación en las instituciones estatales. De esta forma, la sugerencia de un nuevo paradigma para pensar la reforma del Estado impone la redefinición del concepto dominante de autonomía estatal y del modelo de gestión pública a él asociado.

\section{BIBLIOGRAFIA CITADA}

BERGER, Susanne (ed.) (1981), Organizing Interests in Western Europe: Pluralism, Corporatism and the Transformation of Politics. Cambridge, Cabridge University Press.

Callaghy, Thomas M. (1989), "Toward State Capability and Embedded Liberalism in the Third Worls: Lessons for Adjustment", in J. 
M. Nelson (org.), Fragile Coalitions: The Politics of Economic Adjustment. New Brunswick, Transactions Books.

Cawson, Alan (ed.) (1985). Organized Interests and the State: Studies in Meso-Corporatism. Beverly Hills e Londres, Sage Publications.

— (1986), Corporatism and Political Theory. Oxford, Basil Blackwell.

Cohen, Joshua e Rogers, Joel. (1992), "Secondary Associations and Democratic Governance”. Politics \& Society, vol. 20, n4, número especial.

Dinız, Eli (1985), “A Transizao Politica no Brasil: Uma Reavaliazao da Dinámica da Abertura”. Dados, vol. 28, no 3.

(1989a), "Transizao, Partidos e Regimes Politicos: Algumas Considerazoes", en E. Diniz e A. Carmago (orgs.), Continuidade $e$ Mudanza no Brasil da Nova Republica. Sao Paulo, Vertice/Iuperj. (1989b), "Crise Politica, Eleizoes e Dinamica Partidaria no Brasil: Um Balanzo Historico". Dados, vol. 32, nº 3.

- (1991), "Empresariado e Projeto Neoliberal na America Latina: Uma Avaliazao dos Anos 80". Dados, vol. 34, nº 3.

- (1993), "Neoliberalismo e Corporativismo: As Duas Faces do Capitalismo Industrial no Brasil". Revista Brasileira de Ciencias Socias, ano $7, \mathrm{n}^{\mathrm{0}} 20$.

- (1994), "Reformas Economicas e Democracia no Brasil dos Anos 90: As Câmaras Setoriais como Forum de Negociazao". Dados, vol. $37, \mathrm{n}^{\mathbf{0}} 2$.

- e Boschi, Renato. (1994), "Empresariado, Crise do Estado e Democracia no Brasil". Trabajo presentado en el seminario $L a$ Nueva Relación entre el Estado y la Burguesía en América Latina, Buenos Aires, CEDES, 20-21 de mayo, mimeo.

Ducatenzeiler, Graciela e Oxном, Philip. (1994), "Democracia, Autoritarismo y el Problema de la Gobernabilidad en América Latina". Desarrollo Económico, Revista de Ciencias Sociales, vol. $34, n^{\circ} 133$.

Evans, Peter B. (1989), "Predatory, Developmental, and other Apparatuses: A Comparative Political Economy Perspective on the Third World State". Sociological Forum, vol. 4, nº 4.

Faucher, Philippe. (1993), "Politicas de Ajuste ou Erosao do Estado no Brasil?". Dados, vol. 36, no 3 .

Figueiredo, Argelina C. e Limongi, Fernando. (1994), "A Atividade Legislativa no Congresso Pos Constituinte". Novos Estudos Cebrap, $\mathrm{n}^{\mathrm{o}} 38$.

- (1995), "Poderes Legislativos e o Poder do Congresso". Monitor Público, ano $2, \mathrm{n}^{\circ} 5$.

FIORI, José Luis. (1983), "Ajuste, Transizao e Governabilidade: O Enigma Brasileiro", en M. da C. Tavares e J. L. Fiori, Desajuste Global e Modernizazao Conservadora. Rio de Janeiro, Paz e Terra.

FrISCHTAK, Leila L. (1994a), "Governance Capacity and Economic Reform in Developing Countries". World Bank Technical Paper, $\mathrm{n}^{\mathrm{o}}$ 254

- (1994b). "Governance and Economic Reform in Brazil". Trabajo presentado en el seminario O Brasil e o Novo Cenario Mundial, Rio de Janeiro, UERJ, 9-11 de agosto.

GedDes, Bárbara (1994), "Challenging the Conventional Wisdom". Journal of Democracy, vol. 5, $\mathrm{n}^{-} 4$.

GranovetTER, Mark (1985), "Economic Action and Social Structure: The Problem of Embeddedness". American Journal of Sociology, vol. $91, \mathrm{n}^{\mathrm{o}} 3$.

GrindLe, Marilee S. y Thomas, John W. (1991), "Policymakers, Policy Choices and Policy Outcomes: Political Economy of Reform in Developing Countries", en D. Perkins e M. Roemer (eds), Reforming Economic Systems in Developing Countries. Boston, Harvard University Press.
- (1991). Public Choices and Policy Change. The Political Economuy of Reform in Developing Countries. Baltimore e Londres, The Johns Hopkins University Press.

HAGgard, Stephen. (1995). “A Reforma do Estado na America Latina”. Trabajo presentado en la Conferencia Anual sobre Desenvolvimento na America Latina e Caribe (ABCL-LAC), Rio de Janeiro, World Bank, 12-13 de junho, mimeo.

— e Kaufman, Robert. (1993), "O Estado no Processo de Iniciazao e de Consolidazao de Reformas Orientadas para o Mercado", en L. Sola (org.), Estado, Mercado e Democracia: Politica e Economia Comparada. Sao Paulo, Paz e Terra.

- (1994), "The Challenges of Consolidation". Journal of Democracy, vol. $5 \mathrm{n}^{\circ} 4$.

Huntington, Samuel P. (1968). Political Order in Changing Societies. New Haven e Londres, Yale University Press.

— (1975), "The United States", in M. Crozier, S. P. Huntington e J. Watanuki, The Crisis of Democracy, Report on the Governability of Democracies to the Thilateral Commission. Nova Iorque, New York University Press.

Kaufman, Robert K. (1985). "Democratic and Authoritarian Responses to the Debt Issue: Argentina, Brazil and Mexico". International Organization, $\mathrm{n}^{\mathrm{Q}} 39$.

- (1989). "The Politics of Economic Adjustment Policy in Argentina, Brazil and Mexico: Experiences in the 1980s and Challenges for the Future". Policy Sciences, vol. 22, nos. 3-4.

Lamounier, Bolivar. (1991). Depois da transizao, Democracia e Eleizoes no Governo Collor. Sao Paulo, Edizoes Loyola.

- (1992). "Estrutura Institucional e Governabilidade na Decada de 1990", en J. P. dos Reis Velloso (coord.), O Brasil e as Reformas Politicas. Rio de Janeiro, Jose Olympio Editora.

- Nohelen, Dieter (orgs.) (1993), Presidencialismo ou Parlamentarismo, Perspectivas sobre a Reorganizazao Intitucional Brasileira. Sao Paulo, Edizoes Loyola/IDESP.

LEFTWICH, Adrian (1993), "Governance, Democracy and Development in the Third World". Third World Quarterly, vol. 14, no 3.

Lembruch, Gerhard e Schmitter, Philippe C. (eds.) (1992), Patterns of corporatist Policy-Making. Londres, Sage Publications.

LOCKE, Richard M. (1995), Remaking the Italian Economy, Policy Failures and Local Successes in the Contemporary Polity. Ph. D. Thesis, Cornell University Press.

Malloy, James M. (1993), "Politica Economica e o Problema da Governabilidades Democratica nos Andes Centrais", in L. Sola (org.), Estado, Mercado e Democracia. Sao Paulo, Paz e Terra.

Maravall, José María (1994), "The Myth of the Authoritarian Advantage". Journal of Democracy, vol. 5, no 4.

Martins, Luciano, (1994), "Instabilidades Politica e Governabilidad na Construzao Democratica", in J. P. dos Reis Velloso (coord.), Governabilidades, Sistema Politico e Violencia Urbana. Rio de Janeiro Jose Olympio Editora.

Melo, Marcus Andre B. C. de. (1994), "Ingovernabilidade: Desagregando o Argumento." Trabajo presentado en el seminario Governabilidade e Pobreza, Iuperj/urbandata/Universidade de Toronto/Fundazao Ford, Rio de Janeiro, 29 de junho- $1^{\circ}$ de julho, mimeo.

Montelro, Jorge Vianna, (1994) “Resultados versus Processos”. Estrategia Macroeconomica, vol. 2, nº 36 .

- (1995a), "O Poder de Propor: Ou, a Economia das Medidas Provisiorias". Revista de Administrazao Publica, vol. 29, no 4.

- (1995b), "Separazao de Poderes e Estabilidade Constitucional". Trabajo presentado en el seminario Estrategia e Estado Brasileiro, II 
Encontro Nacional de Estudos Estrategicos, Sao Paulo, NAIPPE/USP, 16-18 de agosto.

- (1995c), "Uma Vez Mais, Esta em Pauta o Sentido da Constituizao". Estrategia Macroeconomica, vol. 3, nํ⒍

- (1995d), "Projeto de Mecanismos Institucionais", mimeo.

O'Connor, J. (1973), The Fiscal Crisis of the State. Nova Iorque, St. Martin Press.

O’Donnell, Guillermo, (1991), "Democracia Delegativa?" Novos Estudos Cebrap, $\mathrm{n}^{\circ} 31$.

- (1993), "On the State, Democratization and some Conceptual Problems: A Latin American View with Glances at some Postcommunist Countries", in L. Whitehead (ed), Economic Liberalization and Democratization: Explorations of the Linkages, World Development (número especial). Nova Iorque, Pergamon Press.

Olson, Marcur. (1982), The Rise and Decline of Nations. New Haven, Yale University Press.

PEssanha, Charles (1993), "Notas sobre as Relazoes entre Executivo e Legislativo no Brasil:1964-1992" Trabalho apresentado no XVII Encontro Anual da Anpocs, Caxambu, MG, 22-25 de ouubro, mimeo.

REIS, Fabio Wanderley, (1989), "Estado, Economia, Etica, Interesses: Para a Construzao Democratica do Brasil". Planejamento e Politicas Publicas, $\mathrm{n}^{\circ} \mathrm{1}$.

— (1993), "Estado Liberal, Projeto Nacional, Questao Social". Planejamento e Politicas Publicas, $\mathrm{n}^{\circ} \mathrm{9}$.

- (1994a), "Notas sobre a Reforma do Estado". Revista do Servizo Publico, año 45, vol. 118, nº 3 .

— (1994b), "Governabilidade e Instituizoes Politicas", in J. P. dos Reis Velloso (coord), Governabilidade, Sistema Politico e Violencia Urbana. Rio de Janeiro, Jose Olympio Editora.

— (1995), "Governabilidade, Instituizoes e Partidos". Novos Estudos Cebrap, $\mathrm{n}^{\mathrm{0}} 41$.

Remmer, Karen L. (1986), "The Politics of Economic Stabilization: IMF Stand by Programs in Latin America, 1954 -1984" Comparative Politics, vol. $19, \mathrm{n}^{\circ} 1$.

- (1990), "Democracy and Economic Crisis: The Latin America Experience". World Politics, vol. 42, n 3 .
SANTOS, Wanderley Guilherme Dos. (1992), "Fronteiras do Estado Minimo: Indicazoes sobre o Hibrido Institucional Brasileiro", in J. P. dos Reis Velloso (coord.), O Brasil e as Reformas Politicas. Rio de Janeiro, Jose Olympio Editora.

- (1993), Razoes da Desordem. Rio de Janeiro, Rocco.

- (1994), "Democracia contrafactual ou Estado Efetivo?", in J. P. dos Reis Velloso (coord.), Governabilidade, Sistema Politico e Violencia Urbana. Rio de Janeiro, Jose Olympio Editora.

SChimitter, Philippe C. e LembruCH, Gerhard (eds.) (1979), Trends Toward Corporatist Intermediation. Beverly Hills e Londres, Sage Publications.

Silva, Eduardo. (1993), "Capitalist Coalitions, the State and Neoliberal Economic Restructuring, Chile 1973-88” World Politics, nº 45.

SkIDMORE, Thomas. (1977), "The Politics of Economic Stabilization in Postwar Latin America", in J. Malloy (ed.). Authoritarianism and Corporatism in Latin America, Pittsburg, University of Pittsburg Press.

Sola, Lourdes. (1993), "Estado, Transformazao Economica e Democratizazao no Brasil", in L. Sola (org.), Estado, Mercado e Democracia. Sao Paulo, Paz e Terra.

- (1994a), "The State, Structural Reform and Democratization in Brazil", in W. C. Smith, C. H. Acuña e E. A. Gamarra (eds.), Democracy, Markets and Structural Reform in Latin America: Argentina, Bolivia, Brazil, Chile and Mexico. New Brunswick e Londres, Transaction Publishers.

— (1994b), "Estado, Reforma Fiscal e Governabilidade Democratica: Qual Estado?. Novos Estudos Cebrap, nº 38.

WhiteHEAD, Lawrence. (1989), "Democratic Consolidation and Desinflation: How Linked, How Attainable", in J. M. Nelson (org.), Fragile Coalitions: The Politics of Economic Adjustment. New Brunswick, Transaction Books.

WORLD BANK. (1992a), Governance and Development apud International Bank for Reconstruction and Development.

- (1993), Governance: The World Bank's Experience.

- (1992b), "Adjustment Lending and Mobilization of Private and Public Resources for Growth". Country Economic Departament and Policy and Research Series, $\mathrm{n}^{\circ} 22$.

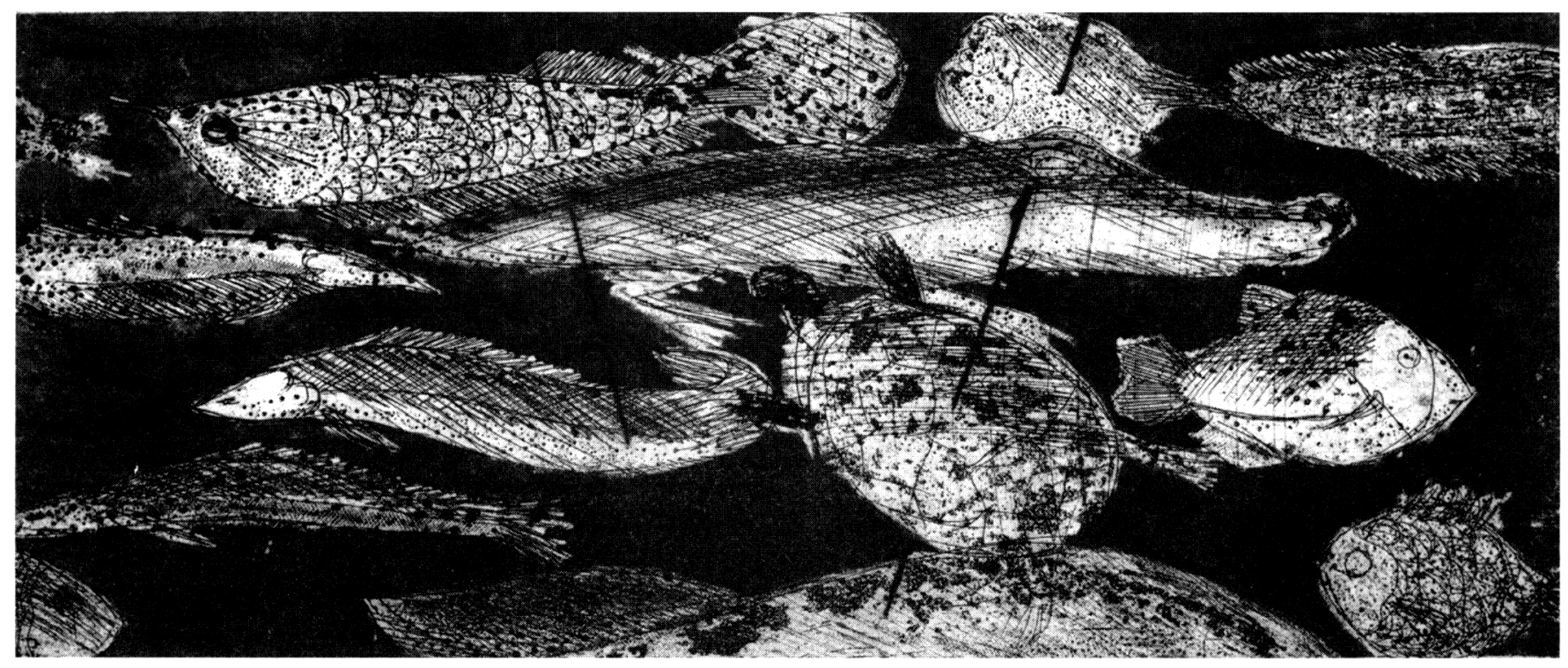




\title{
RESUMEN
}

Las múltiples dimensiones de la crisis brasileña han quedado oscurecidas por su vertiente económica. Por esta razón, la problemática de la gobernabilidad ha sido abordada en la gran mayoría de los estudios desde la óptica del ajuste macroeconómico, centrado en la crisis fiscal del Estado. No obstante, es necesario atender a diversos problemas políticos -tanto a estructuras institucionales como a dinámicas sociales específicas- que inciden sobre el tipo governance que se configura desde el aparato de estado e influyen en el carácter incluyente (democrático o no) de la resolución de la coyuntura de crisis.

\begin{abstract}
The multiple dimensions of brazilean crisis have been covered by its economic aspect. For this reason, the problem of governability has been analyzed in the most of the studies from the perspective of the macroeconomic adjustment, centered in the state fiscal crisis. However, it is necessary to attend the different political problems, in terms of institutional structures and specifical social dynamics, which affect the type governance configured from the state aparatus and which influence the inclusing character (democratic or non) to solve the critical conjucture.
\end{abstract}

